\title{
Transcatheter valve-in-valve implantation in degenerated aortic bioprostheses: are patients with small surgical bioprostheses at higher risk for unfavourable mid-term outcomes?
}

\author{
Clarence Pingpoh $^{1,2}$, Holger Schroefel ${ }^{1,2}$, Tanja Franz ${ }^{1,2}$, Martin Czerny $^{1,2}$, Maximilian Kreibich ${ }^{1,2}$, \\ Martin Moser ${ }^{3}$, Christoph Bode ${ }^{3}$, Friedhelm Beyersdorf ${ }^{1,2}$, Franz-Josef Neumann ${ }^{4}$, Willibald Hochholzer ${ }^{4}$, \\ Matthias Siepe ${ }^{1,2}$ \\ ${ }^{1}$ Department of Cardiovascular Surgery, University Heart Center Freiburg • Bad Krozingen, Bad Krozingen, Germany; ${ }^{2}$ Faculty of Medicine, Albert- \\ Ludwigs-University of Freiburg, Freiburg, Germany; ${ }^{3}$ Department of Cardiology and Angiology I, Heart Center, University of Freiburg, Faculty \\ of Medicine, Freiburg, Germany; ${ }^{4}$ Department of Cardiology and Angiology II, University Heart Center Freiburg-Bad Krozingen, Bad Krozingen, \\ Germany \\ Correspondence to: Clarence Pingpoh, MD. Department of Cardiovascular Surgery, University Heart Center Freiburg · Bad Krozingen, Suedring 15, \\ 79189 Bad Krozingen, Germany. Email: Clarence.Pingpoh@universitaets-herzzentrum.de.
}

\begin{abstract}
Background: To examine outcomes of valve-in-valve (ViV) transcatheter aortic valve implantation (TAVI) according to the inner diameter (ID) of the degenerated aortic valve bioprosthesis.

Methods: We analyzed survival, stroke, permanent pacemaker (PPM) implantation, paravalvular (PV) leakage, acute kidney injury and vascular complications in fifty-nine patients during a ten-year period. Patients were stratified according to the ID of the indwelling degenerated biological aortic valve (true ID $\leq$ and $>20 \mathrm{~mm}$ ). Differences in post-procedural transvalvular gradients and hospital re-admissions were analyzed.

Results: The median age of the small diameter group and large diameter group was eighty-one and eighty years, respectively. Median logistic EuroSCORE I was 23.9\% and 26.2\% and median Society of Thoracic Surgeons (STS) score was $5.7 \%$ and $7.8 \%$ for the small and large groups, respectively. Survival, stroke, PPM implantation, PV leakage, acute kidney injury and vascular complications did not reach any statistically significant difference between both groups. Postprocedural transvalvular gradients differed significantly according to the true ID of the degenerated bioprosthetic valve and consequently of the respective TAVI valve. There was a significant difference with regard to hospital readmissions according to the true ID.

Conclusions: TAVI ViV implantation for aortic bioprostheses with small true IDs of $\leq 20 \mathrm{~mm}$ is associated with comparable mid-term mortality and periprocedural stroke rate compared to implantation into larger bioprostheses. However, the periprocedural and mid-term transvalvular gradients, as well as hospital re-admission rates are significantly higher in the small diameter group.
\end{abstract}

Keywords: Transcatheter valve implantation; aortic valve prosthesis; transvalvular gradient; true inner diameter (true ID); mortality

Submitted Jun 30, 2020. Accepted for publication Nov 09, 2020.

doi: 10.21037/acs-2020-av-fs-0124

View this article at: http://dx.doi.org/10.21037/acs-2020-av-fs-0124

\section{Introduction}

Transcatheter aortic valve implantation valve-in-valve (TAVI ViV) implantation has become common practice for treatment of moderate to high risk patients with a degenerated aortic valve bioprosthesis (1-3). Since there is a trend towards bioprostheses for surgical aortic valve replacement (SAVR), it is expected that the number of transcatheter $\mathrm{ViV}$ procedures will increase (1). Concerns 
have been raised regarding the technical feasibility and in particular the risk of residual transvalvular gradients following this procedure, in particular for small surgical bioprosthesis (1). Patient prosthesis mismatch and small surgical valves [inner diameters (ID) $\leq 20 \mathrm{~mm}$ ] are known risk factors for high post procedural transvalvular gradients which in turn might affect mid-term outcome (1-6). In this study, we evaluated the outcomes of patients undergoing TAVI ViV implantation in a dedicated structural heart disease team.

\section{Methods}

\section{Study population and objectives}

This retrospective study analyzed patients with a history of TAVI ViV implantation at our institution from December 2010 until September 2019. The study was approved by our institutional ethics committee (EK-Freiburg: $555 / 19)$. Patient data were extracted from the institutional database and included the following variables: patient characteristics (e.g., age, sex, height, weight), clinical history (e.g., previous cardiac operation, cardiovascular risk factors), echocardiographic variables (ejection fraction, right ventricular function, valve status and size), pre-TAVI computer tomography analysis (effective orifice area, indexed effective orifice area) and laboratory variables as well as any existing follow-up data. Inclusion criteria were history of SAVR with a bioprosthesis and subsequent TAVI $\mathrm{ViV}$ implantation within the study period at our institution. Using the "ViV Aortic" application (UBQO Limited), key information on the surgically implanted aortic bioprosthesis such as the true ID and the stent diameter were analyzed.

The diagnosis and evaluation of bioprosthetic degeneration was made by echocardiography. The decision for TAVI ViV was made by a dedicated heart team including cardiac surgeons, interventional and non-interventional cardiologists, imaging specialists, and anesthesiologists, and was mainly based on surgical risk according to logistic EuroSCORE I and Society of Thoracic Surgeons (STS) Score as well as the morphology of the aorta and patient-specific factors such as frailty. The access route was either transfemoral or transapical depending on the comorbidities of the patient, with the transfemoral route preferred whenever both ways were possible. Dopplerechocardiography was performed at discharge. We analysed the pre- and post-interventional computed tomography (CT) scans as well as the peri-interventional and follow-up echocardiographic data. Follow-up visits were performed at irregular intervals by local cardiologists or at our outpatient unit.

\section{Parameters analyzed}

We analyzed survival, stroke, permanent pacemaker (PPM) implantation, paravalvular (PV) leakage, acute kidney injury and vascular complications in fifty-nine patients during a ten-year period. Patients were stratified according to ID of the indwelling degenerated biological aortic valve (true ID $\leq$ and $>20 \mathrm{~mm}$ ) (see Figure 1). Differences in postprocedural transvalvular gradients and hospital readmissions were analyzed accordingly. The endpoints are aligned to the Valve Academic Research Consortium (VARC-2) criteria (6).

\section{Statistics}

The median true ID of the cohort was normally distributed around $20 \mathrm{~mm}$. Thus, patients were initially stratified into a small diameter (true ID $\leq 20 \mathrm{~mm}$ ) and a large diameter group (true ID $>20 \mathrm{~mm}$ ). Discrete variables are reported as counts (percentages) and continuous variables as median and quartiles. For discrete variables, we tested differences between groups with the $\chi^{2}$-test or Fisher's exact test when expected cell sizes were less than five. The Mann Whitney $\mathrm{U}$ test was used for comparison of non-Gaussian continuous variables. In the two-sided test, a $\mathrm{P}$ value $<0.05$ was regarded as significant. For the survival analysis, the outcome of interest was 'Alive' or 'Dead' (whichever occurred within the observation period). The Kaplan-Meier method was used to estimate overall survival and the log rank test to compare the survival distributions of the two groups. A significance level of $<0.05$ was used. Statistical analyses were run with Stata statistical software package by StataCorp.

\section{Results}

\section{Patient characteristics}

Patient characteristics are shown in Table 1. From 2010 to 2019,59 patients meeting the inclusion criteria were identified. Five-year follow-up was complete within our study period. Baseline characteristics are summarized in Table 1. Median age in the small and large diameter groups was eighty-one and eighty years, respectively. The median body mass indices in the two groups were 25.5 (small diameter group) and 25.6 (large diameter group). Median logistic EuroSCORE I was $23.9 \%$ for the small diameter group and $26.2 \%$ for the large diameter group. The median 
Surgical Bioprostheses SJM Epic, Carpentier Edwards, Medtronic Freestyle, Sorin Mitroflow, Homograft, Medtronic -Mosaic, -Hancock Jenavalve,

Transcatheter bioprostheses CoreValve-Evolut, -Evolut R, Edwards-Sapien, -Sapiens XT, Symetis Accurate

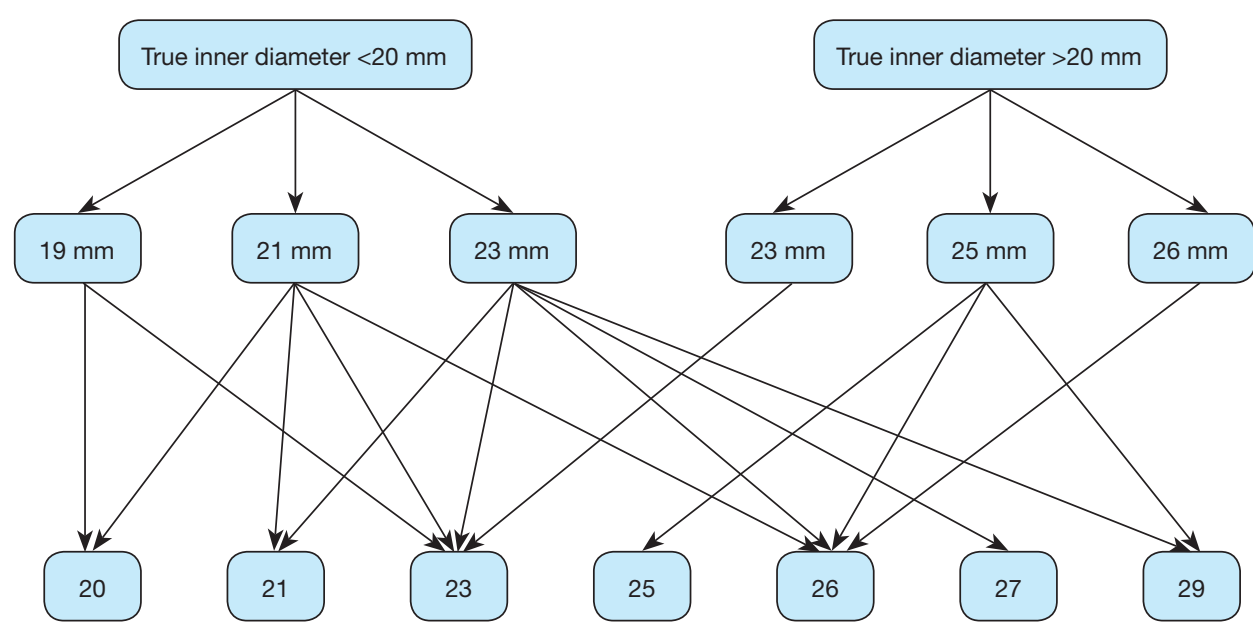

Figure 1 Surgical and transcatheter valves before TAVI ViV procedure. TAVI, transcatheter aortic valve implantation; ViV, valve-in-valve.

STS score was $5.7 \%$ and $7.8 \%$ for the small and large diameter groups, respectively. Prior to TAVI ViV, more than $60 \%$ of the patients in both groups presented with angina pectoris (CCS III/IV) and/or symptoms pertaining to a New York Heart Association (NYHA) class III/IV.

\section{Pre-TAVI ViV echocardiography and computer tomography}

Table 2 shows differences in preintervention echocardiography and computer tomography. The median mean transvalvular gradient (Dpmean) before the procedure was 43.5 and $39 \mathrm{mmHg}$ in small and large diameter groups, respectively (Table 2). Median pre-interventional maximum transvalvular velocity (Vmax) was 3.3 and $3.0 \mathrm{~m} / \mathrm{s}$ for the small and large diameter groups, respectively. No difference between the groups was observed with regards to the pre-TAVI ViV left ventricular ejection fraction. The median effective orifice area index (EOAI) prior to the TAVI $\mathrm{ViV}$ procedure was 1.1 for the small diameter group and 1.2 for the large diameter group. EOAI based on the true ID showed no severe patient-prosthesis-mismatch (ppm) in both groups before the procedure.

\section{Post-TAVI ViV echocardiography and outcome}

Table 3 shows differences in the outcomes between both groups. Median postprocedural Vmax was 2.4 and $1.8 \mathrm{~m} / \mathrm{s}$ in the small and large diameter groups, respectively. Median
Dpmean was $25.5 \mathrm{mmHg}$ in the small diameter group and $16 \mathrm{mmHg}$ in the large diameter group (Figure 2 and Table 3). More than $90 \%$ of the patients in both groups showed no peri-interventional decline of left ventricular ejection fraction. The right ventricular function in both groups was normal or mildly impaired. Mild PV leakage was present in $39 \%(11 / 28)$ and $40 \%(8 / 20)$ of the patients in the small and large diameter groups, respectively. Only $7 \%(2 / 28)$ patients (small diameter group) had moderate PV leakage. The high rates of PV leakage seem overrepresented due to the small cohort. Hospital readmission occurred in thirteen patients; 39\% (11/28) from the small diameter group and $10 \%(2 / 20)$ from the large diameter group. Only one patient (large diameter group) had a post-procedural stroke. All patients who received a PPM implantation in this cohort were from the small diameter group $(14 \%, 4 / 28, \mathrm{P}=0.077)$. Twelve patients died during follow-up; eight from the small diameter group and four from the large diameter group. The difference in the survival curves of patients in these cohorts was not statistically significant (Figure 3) (Log rank $\mathrm{P}=0.73$ ). The overall survival after eighty months was $78 \%$ and remained unchanged till the end of the observation period (Figure 3).

\section{Discussion}

\section{Patient characteristics}

Transcatheter ViV implantation is gradually becoming 


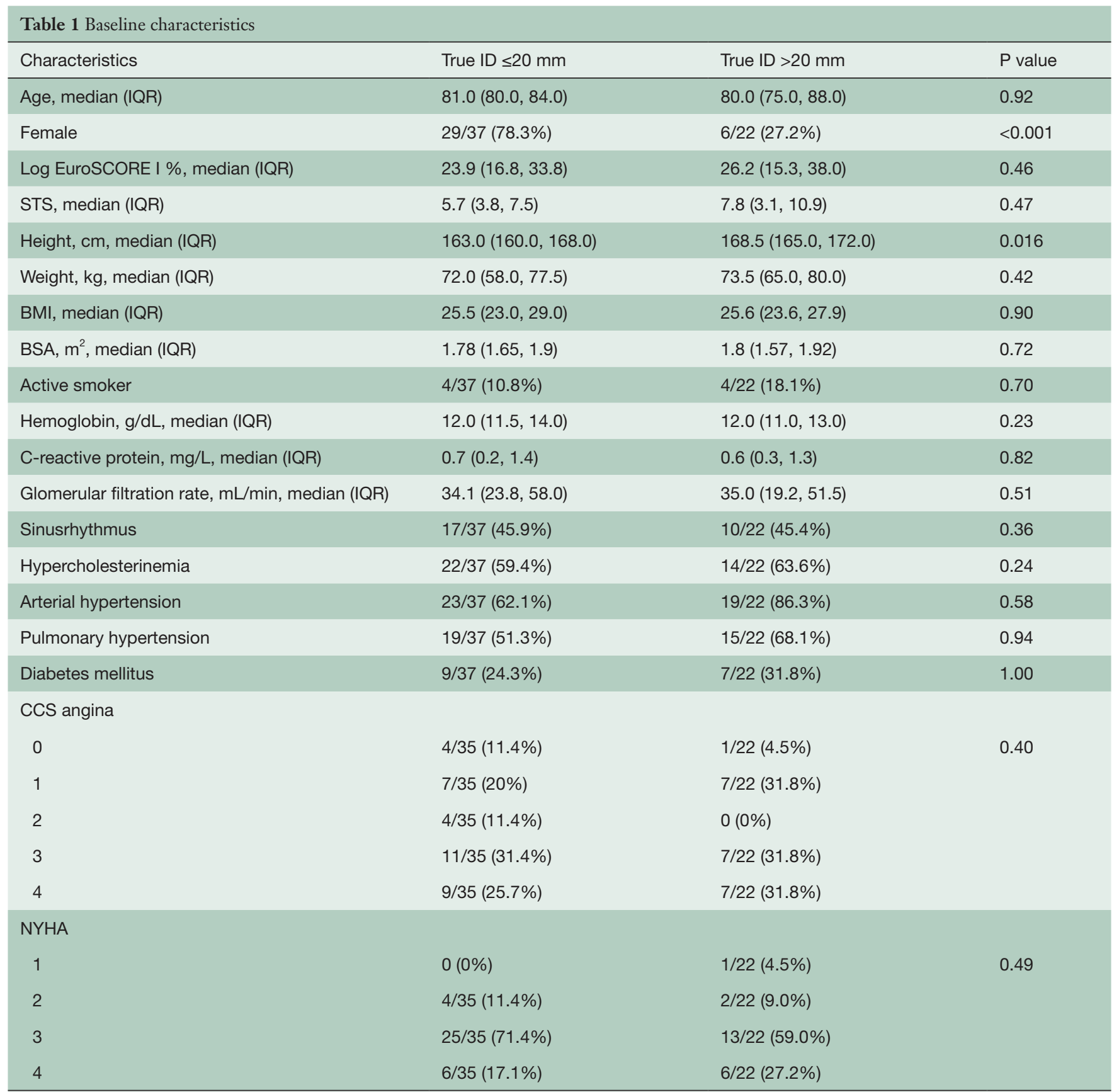

Data are presented as median (first quartile; third quartile) or as number (\%); GFR, glomerular filtration rate; BMI, body mass index; STS, Society of Thoracic Surgeons; CCS, Canadian Cardiovascular Society; NYHA, New York Heart Association.

common practice in treating degenerated aortic bioprostheses in moderate to high risk patients. The patients in the systematic review and meta-analysis by Phan $e t a l$. (1) had a mean age of 77.5 years and the mean logistic EUROSCORE I was thirty-one. Erlebach et al. (7) also showed in their work that patients receiving TAVI
ViV were significantly older, had a higher mean logistic EuroSCORE and exhibited a lower mean left ventricular ejection fraction than patients receiving surgical prosthesis replacement. This is similar to our patient population with a median age of eighty-one as well as a median logistic EUROSCORE of $25 \%$. These as well 


\begin{tabular}{|c|c|c|c|}
\hline Variables & True ID $\leq 20$ mm & True ID >20 mm & $P$ value \\
\hline \multicolumn{4}{|l|}{ Left ventricular function } \\
\hline$E F>50 \%$ & $26 / 37(70.2 \%)$ & $13 / 21(61.9 \%)$ & \multirow[t]{2}{*}{0.78} \\
\hline EF $40-50 \%$ & $6 / 37(16.2 \%)$ & $4 / 21(19.0 \%)$ & \\
\hline \multicolumn{4}{|l|}{ Right ventricular function } \\
\hline Normal & $30 / 37(81 \%)$ & $18 / 21(85.7 \%)$ & \multirow[t]{3}{*}{0.35} \\
\hline Mildly impaired & $2 / 37(5.4 \%)$ & $3 / 21(14.2 \%)$ & \\
\hline Moderately impaired & $2 / 37(5.4 \%)$ & $0(0 \%)$ & \\
\hline Dpmean, mmHg, median (IQR) & $43.5(30.0,51.5)$ & $39.0(17.0,46.0)$ & 0.16 \\
\hline Vmax, m/s, median (IQR) & $3.3(2.5,4.0)$ & $3.0(2.0,3.2)$ & 0.020 \\
\hline True ID, mm, median (IQR) & $18.5(17.0,19.0)$ & $21.5(21.0,23.0)$ & $<0.001$ \\
\hline Stent ID, mm, median (IQR) & $19.0(18.0,20.5)$ & $23.0(22.0,23.0)$ & $<0.001$ \\
\hline EOA-true ID, median (IQR) & $1.1(1.0,1.3)$ & $1.2(1.1,1.5)$ & 0.017 \\
\hline
\end{tabular}

Data are presented as median (first quartile; third quartile) or as number (\%); EF, ejection fraction; Dpmax, maximum transvalvular gradient; Dpmean, mean transvalvular gradient; Vmax, maximum transvalvular velocity; ID, inner diameter; EOAI, effective orifice area index.

as other current studies $(2,3)$ suggest that TAVI ViV is the treatment of choice for advanced age patients with a moderate to high surgical risk, and the guidelines support this treatment strategy $(4,5)$.

\section{Patient-prosthesis-mismatch}

In 2014, Bapat and colleagues (8) studied the effect of the internal diameter of a bioprosthetic valve and its implications for the $\mathrm{ViV}$ procedure using valve models. Their work provided a chart which is the basis of the values for the true IDs in this study. True ID is defined as the ID of the inflow of the surgical heart valve. The median true ID of this cohort was normally distributed around $20 \mathrm{~mm}$ : median true ID was $18.5 \mathrm{~mm}$ for the small diameter group and $21.5 \mathrm{~mm}$ for the large diameter group.

The median effective orifice area index prior to the TAVI ViV procedure in our patient cohort was 1.1 and 1.2 in the small and large diameter groups respectively. This is a marked difference to previous studies (9-17), which reported a higher rate of patient-prosthesis-mismatch with similarly small true IDs. Despite a low rate of patient prosthesis mismatch in our cohort, we observed relatively high transvalvular gradients in the surgical bioprostheses before TAVI ViV. This may be suggestive of a hemodynamic mismatch before the procedure.

Okuno et al. (18). report in their study that selfexpanding valves were associated with a lower frequency of PPM implantation compared to balloon expanding valves irrespective of annulus area in native aortic annuli. In our study, we saw no difference in the occurrence of PPM with respect to the implanted valves.

The hemodynamic effects of small valve sizes, patientprosthesis mismatch or residual transvalvular gradients following TAVI ViV procedure on survival have been investigated by several studies (2,9-17) and there is a tendency towards assuming that these gradients might lead to worse outcomes (12-21). This question could not be definitively answered in this study and although numerically more patients with a true ID of $\leq 20 \mathrm{~mm}$ died, this trend did not reach any statistically significant difference in the fiveyear mortality. 


\begin{tabular}{|c|c|c|c|}
\hline Variables & True ID $\leq 20 \mathrm{~mm}$ & True ID >20 mm & $P$ value \\
\hline \multicolumn{4}{|l|}{ Left ventricular function } \\
\hline$E F>50 \%$ & $27 / 37(72.9 \%)$ & $12 / 21(57.1 \%)$ & \multirow[t]{4}{*}{0.58} \\
\hline EF $40-50 \%$ & $5 / 37(13.5 \%)$ & $5 / 21(23.8 \%)$ & \\
\hline EF $35-40 \%$ & $4 / 37(10.8 \%)$ & $3 / 21(14.3 \%)$ & \\
\hline$E F<35 \%$ & $1 / 37(2.7 \%)$ & $1 / 21(4.7 \%)$ & \\
\hline \multicolumn{4}{|l|}{ Right ventricular function } \\
\hline Normal & $32 / 35(91.4 \%)$ & $18 / 20(90 \%)$ & \multirow[t]{3}{*}{0.76} \\
\hline Mildly impaired & $2 / 35(5.7 \%)$ & $2 / 20(10 \%)$ & \\
\hline Moderately impaired & $1 / 35(2.9 \%)$ & $0(0 \%)$ & \\
\hline Dpmax, mmHg, median (IQR) & $44.5(24.5,61.0)$ & $30.0(19.0,38.0)$ & 0.01 \\
\hline Dpmean, mmHg, median (IQR) & $25.5(13.0,33.5)$ & $16.0(11.0,20.0)$ & 0.03 \\
\hline Vmax, m/s, median (IQR) & $2.4(1.9,3.0)$ & $1.8(1.5,2.1)$ & 0.003 \\
\hline Glomerular filtration rate, $\mathrm{mL} / \mathrm{min}$, median (IQR) & $39.9(28.9,62.0)$ & $43.5(21.4,64.4)$ & 0.95 \\
\hline 5-year mortality & $8 / 37(21.6 \%)$ & $4 / 22(18.1 \%)$ & 0.75 \\
\hline Stroke & $0(0 \%)$ & $1 / 20(5 \%)$ & 0.23 \\
\hline \multicolumn{4}{|l|}{ Paravalvular leak } \\
\hline None & $15 / 28(53.5 \%)$ & $12 / 20(60 \%)$ & \multirow[t]{3}{*}{0.68} \\
\hline Mild & $11 / 28(39.3 \%)$ & $8 / 20(40 \%)$ & \\
\hline Moderate & $2 / 28(7.1 \%)$ & $0(0 \%)$ & \\
\hline Hospital readmission & $11 / 28(39.3 \%)$ & $2 / 20(10 \%)$ & 0.024 \\
\hline Vascular complication & $3 / 28(10.7 \%)$ & $1 / 20(5 \%)$ & 0.48 \\
\hline Permanent pacemaker & $4 / 28(14.3 \%)$ & $0(0 \%)$ & 0.08 \\
\hline Acute kidney injury & $6 / 24(25 \%)$ & $2 / 14(14.3 \%)$ & 0.43 \\
\hline Composite endpoint (survival and hospital readmission) & $14 / 29(48.2 \%)$ & 4/21 (19\%) & 0.034 \\
\hline $\begin{array}{l}\text { Composite in-hospital endpoint (stroke/pacemaker, acute } \\
\text { kidney failure) }\end{array}$ & 9/27 (33.3\%) & $3 / 20(15 \%)$ & 0.15 \\
\hline
\end{tabular}

Data are presented as median (first quartile; third quartile) or as number (\%); EF, ejection fraction; Dpmax, maximum transvalvular gradient; Dpmean, mean transvalvular gradient; Vmax, maximum transvalvular velocity.

\section{Follow-up and outcome}

The idea of smaller ID and poor hemodynamic values correlating with a negative outcome seems to be implied in current studies (12-21), even though complete followup data for these patients are lacking. In our study, we see that the postoperative transvalvular values (Dpmean, Dpmax and Vmax) are significantly worse in the small ID group. Our follow-up over eighty months was complete and we observed that overall survival was good but hospital readmissions were higher in the small diameter group $(11 / 28)$ than in the large diameter group (2/20). The stroke and PPM rates were relatively low with respect to the total population. All four patients with a PPM had previously received a stented surgical bioprosthesis (Carpentier Edwards Magna Ease; $\mathrm{n}=3$ and Mitroflow; $\mathrm{n}=1$ ) with the true ID varying from $17-19 \mathrm{~mm}$. The transcatheter valve 


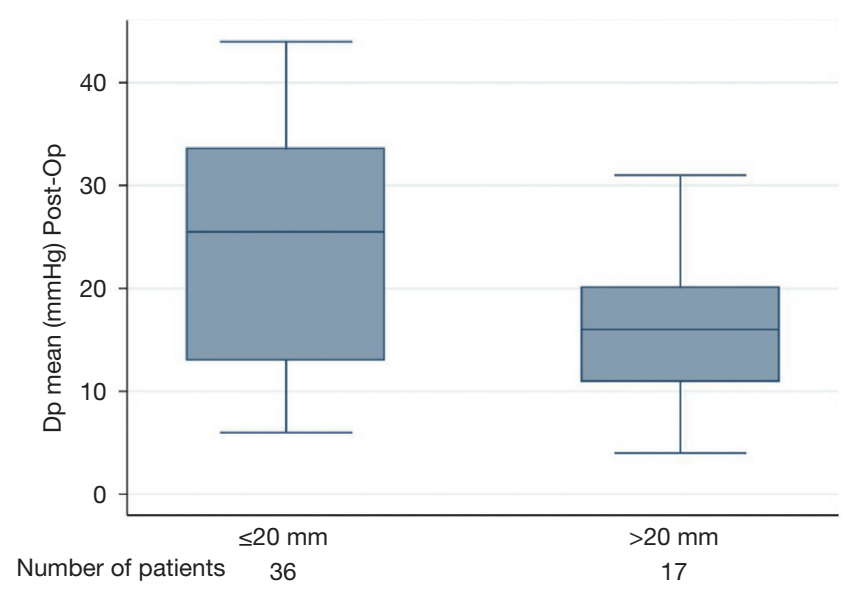

Figure 2 Mean postoperative transvalvular gradients in patients with true $\mathrm{ID} \leq 20 \mathrm{~mm}$ and $>20 \mathrm{~mm}(\mathrm{P}=0.03)$.

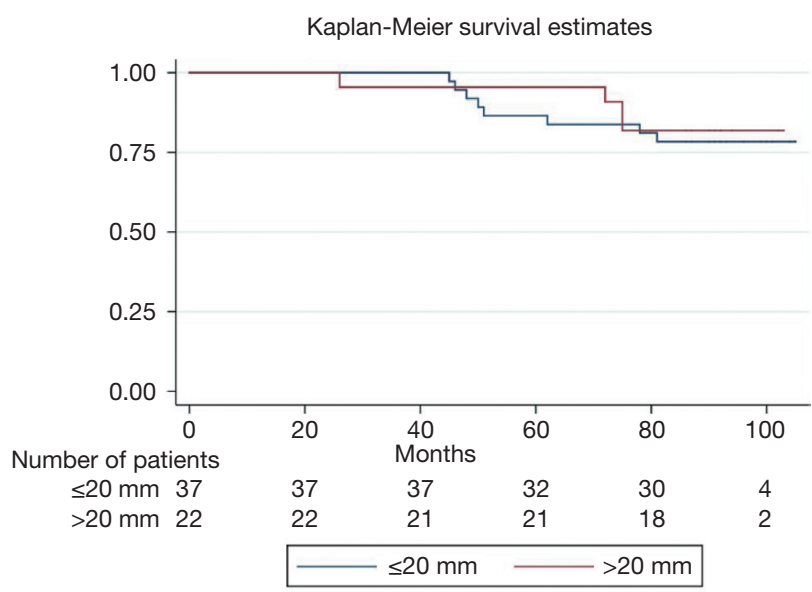

Figure 3 Kaplan Meier survival estimates $(\mathrm{P}=0.75)$.

was implanted in all cases without any prior dilatation of the surgical prosthetic valve. The overall survival after eighty months was $78 \%$ and remained unchanged until the end of the observation period. We observed twelve deaths during follow-up; eight in the small diameter group and four in the large diameter group. The median age of our cohort was eighty years. Most patients died out of hospital and we cannot safely say if the cause of death was cardiovascular or otherwise. This difference in survival did not reach statistical significance. Bleiziffer et al. (2) and Seiffert et al. (3) report similar results with elevated gradients after TAVI $\mathrm{ViV}$, which were not associated with poorer short-term survival and clinical outcomes. However, residual stenosis remains a problem, particularly in smaller bioprostheses and small true IDs. All readmissions were those recorded at our heart center, hence were due to cardiovascular reasons. Four were for pacemaker checkups, two were for control of the PV leakage at the time of implantation and the others were routine follow up echocardiography. From the available data we cannot be sure if the higher residual gradients for the small group resulted in higher rates of readmissions since we did not record the reasons for readmissions. Nevertheless, an association between both findings with higher morbidity due to higher gradients is possible and likely.

Rayol et al. (22) reported that PPM is a frequent and relevant hemodynamic complication of SAVR. Surgeons should prospectively calculate the predicted indexed EOA of the prosthesis and patient's body surface area (BSA) and hence plan their surgical procedure adequately; either with an aortic root enlargement (Nikcs-Nunez or Manougian technique) or with a rapid deployment surgical valve to avoid PPM.

\section{Strengths and limitations}

This analysis with a relatively small patient number is retrospective with all inherent limitations. There was no CORE Lab echocardiography standard before and after the procedure although the examination was consistently performed by experienced echocardiographers.

\section{Conclusions}

TAVI ViV in bioprostheses with a small true ID of $\leq 20 \mathrm{~mm}$ produces similar mid-term results in terms of mortality and periprocedural stroke rate as compared to larger prostheses. The periprocedural and mid-term transvalvular gradients, as well as hospital readmission rates are significantly higher in the small diameter group. The five-year mortality between both groups did not reach any statistically significant difference although numerically more patients with a true ID of $\leq 20 \mathrm{~mm}$ died during follow-up.

\section{Acknowledgments}

Funding: This trial was supported by the University Heart Center Freiburg · Bad Krozingen.

\section{Footnote}

Conflicts of Interest: FJN reports grants and fees to the 
institution from Daiichi Sankyo, Astra Zeneca, SanofiAventis, Bayer, The Medicines Company, Bristol, Novartis, Roche, Boston Scientific, Biotronik, Medtronic, Edwards Lifesciences and Ferrer. The other authors have no conflicts of interest to declare.

Open Access Statement: This is an Open Access article distributed in accordance with the Creative Commons Attribution-NonCommercial-NoDerivs 4.0 International License (CC BY-NC-ND 4.0), which permits the noncommercial replication and distribution of the article with the strict proviso that no changes or edits are made and the original work is properly cited (including links to both the formal publication through the relevant DOI and the license). See: https://creativecommons.org/licenses/by-nc-nd/4.0/.

\section{References}

1. Phan K, Zhao DF, Wang N, et al. Transcatheter valvein-valve implantation versus reoperative conventional aortic valve replacement: a systematic review. J Thorac Dis 2016;8:E83-E93.

2. Bleiziffer S, Erlebach $M$, Simonato M, et al. Incidence, predictors and clinical outcomes of residual stenosis after aortic valve-in-valve. Heart 2018;104:828-34.

3. Seiffert M, Treede H, Schofer J, et al. Matched comparison of next- and early-generation balloon-expandable transcatheter heart valve implantations in failed surgical aortic bioprostheses. EuroIntervention 2018;14:e397-e404.

4. Falk V, Baumgartner H, Bax JJ, et al. 2017 ESC/EACTS Guidelines for the management of valvular heart disease. Eur J Cardiothorac Surg 2017;52:616-64.

5. Kappetein AP, Head SJ, Généreux P, et al. Updated standardized endpoint definitions for transcatheter aortic valve implantation: the Valve Academic Research Consortium-2 consensus document (VARC-2). Eur J Cardiothorac Surg 2012;42:S45-S60.

6. Kappetein AP, Head SJ, Généreux P, et al. Valve Academic Research Consortium-2. Updated standardized endpoint definitions for transcatheter aortic valve implantation: the Valve Academic Research Consortium-2 consensus document. J Thorac Cardiovasc Surg 2013;145:6-23.

7. Erlebach M, Wottke M, Deutsch MA, et al. Redo aortic valve surgery versus transcatheter valve-in-valve implantation for failing surgical bioprosthetic valves: consecutive patients in a single-center setting. J Thorac Dis 2015;7:1494-500.

8. Bapat VN, Attia R, Thomas M. Effect of valve design on the stent internal diameter of a bioprosthetic valve: a concept of true internal diameter and its implications for the valve-in-valve procedure. JACC Cardiovasc Interv 2014;7:115-27.

9. Dvir D, Webb J, Brecker S, et al. Transcatheteraortic valve replacement for degenerative bioprosthetic surgical valves: results from the global valve-in-valve registry. Circulation 2012;126:2335-44.

10. Pibarot P, Weissman NJ, Stewart WJ, et al. Incidence and sequelae of prosthesis- patient mismatch in transcatheter versus surgical valve replacement in high-risk patients with severe aortic stenosis: a partner trial cohort-a analysis. J Am Coll Cardiol 2014;64:1323-34.

11. Dvir D, Webb JG, Bleiziffer S, et al. Transcatheter aortic valve implantation in failed bioprosthetic surgical valves. JAMA 2014;312:162-70.

12. Lopez S, Mathieu P, Pibarot P, et al. Does the use of stentless aortic valves in a subcoronary position prevent patient-prosthesis mismatch for small aortic annulus? J Card Surg 2008;23:331-5.

13. Pibarot P, Dumesnil JG. Hemodynamic and clinical impact of prosthesis-patient mismatch in aortic valve position and its prevention. J Am Coll Cardiol 2000;36:1131-41.

14. Pibarot P, Dumesnil JG, Lemieux M, et al. Impact of prosthesis-patient mismatch on hemodynamic and symptomatic status, morbidity and mortality after aortic valve replacement with a bioprosthetic heart valve. J Heart Valve Dis 1998;7:211-8.

15. Head SJ, Mokhles MM, Osnabrugge RL, et al. The impact of prosthesis-patient mismatch on long-term survival after aortic valve replacement: a systematic review and meta-analysis of 34 observational studies comprising 27186 patients with 133141 patient-years. Eur Heart J 2012;33:1518-29.

16. Poulin F, Yingchoncharoen T, Wilson WM, et al. Impact of prosthesis-patient mismatch on left Ventricular myocardial mechanics after transcatheter aortic Valve replacement. J Am Heart Assoc 2016;5:e002866.

17. Mooney J, Sellers SL, Blanke P, et al. Prosthesis-patient mismatch downgrades frequency and severity, and demonstrates no association with adverse outcomes after transcatheter aortic valve replacement. JACC Cardiovasc Interv 2017;10:1578-87.

18. Okuno T, Khan F, Asami M, et al Prosthesis-Patient Mismatch Following Transcatheter Aortic Valve Replacement with Supra-Annular and Intra-Annular Prostheses. JACC Cardiovasc Interv 2019;12:2173-82.

19. Simonato M, Webb J, Kornowski R, et al. Transcatheter 
Replacement of Failed Bioprosthetic Valves: Large Multicenter Assessment of the Effect of Implantation Depth on Hemodynamics After Aortic Valve-in-Valve. Circ Cardiovasc Interv 2016;9:e003651.

20. Johansen P, Engholt H, Tang M, et al. Fracturing mechanics before valve-in-valve therapy of small aortic bioprosthetic heart valves. EuroIntervention 2017;13:e1026-e1031.

21. Leon MB, Piazza N, Nikolsky E, et al. Standardized

Cite this article as: Pingpoh C, Schroefel H, Franz T, Czerny M, Kreibich M, Moser M, Bode C, Beyersdorf F, Neumann FJ, Hochholzer W, Siepe M. Transcatheter valve-in-valve implantation in degenerated aortic bioprostheses: are patients with small surgical bioprostheses at higher risk for unfavourable mid-term outcomes? Ann Cardiothorac Surg 2020;9(6):478-486. doi: 10.21037/acs-2020-av-fs-0124 endpoint definitions for transcatheter aortic valve implantation clinical trials: a consensus report from the valve academic research consortium. J Am Coll Cardiol 2011;57:253-69.

22. Rayol SDC, Sá MPBO, Cavalcanti LRP, et al. ProsthesisPatient Mismatch after Surgical Aortic Valve Replacement: Neither Uncommon nor Harmless. Braz J Cardiovasc Surg 2019 34:361-5. 